\title{
The secret masters of the planet reveal their secrets
}

\author{
Peter Nick
}

Published online: 7 February 2015

(C) Springer-Verlag Wien 2015

Evolution is generally visualised in the form of trees, where so-called primitive organisms are depicted near the trunk, whereas the upper branches are reserved for the so-called advanced life forms (including the "higher" primate Homo sapiens). Although convenient to show evolutionary changes, these trees have a drawback - the fact that a life form is simple does not at all mean that it is "primitive". It has adapted to a specific ecological niche over a very long time, and it is, thus, as perfect as any other life form from the upper branches. This case holds especially true for the unicellular algae that are considered as "lower plants", from which the more conspicuous terrestrial "higher plants" have derived. It is often ignored that these "lower plants" have developed sophisticated adaptations to even the most unusual environments and that, by this degree of sophistication, they were able to conquer virtually any illuminated niche on this planet. By their wide distribution and versatility, they can easily keep pace and even excel all the higher plants that later have derived from them. Two contributions in the current issue address the astonishing secrets of these secret masters of our planet:

The conquest of solid land was a crucial event for evolution as a whole and required numerous adaptations that had to develop in concert to be effective. If one wants to avoid unlikely scenarios, where such novel changes happen by coincidence, one has to search for preadaptations in related taxa that might, under the changed circumstances, acquire a novel functional significance. In the context of the first land plants, the sister clades of Zygnematophyceae and Coleochaetophyceae are interesting. It has been proposed that physiological pread-

P. Nick $(\bowtie)$

Botanical Institute, Karlsruhe Institute of Technology,

Karlsruhe, Germany

e-mail: peter.nick@kit.edu aptation of aero-terrestrial streptophyte algae might have prepared the decisive step to land life. The ultrastructure of these algae has been characterised in great detail, including the details of cell division for the genus Zygnema (Bakker and Lokhorts 1987), which allows to interpret physiological adaptations of cellular organisation in a functional context. The work by Herburger et al. (2015) in the current issue investigates such preadaptations in great morphological and physiological detail for different strains of Zygnema. While the physiological adaptations to drought and freezing have been characterised for Arctic or Antarctic species of this genus, the current work addresses the adaptations of Alpine species, where, due to higher altitude, considerable stress by UV-B has to be compensated, which was one of the major challenges for the first land plants. Authors show that a specific population of storage cells, the pre-akinetes, seem to be crucial for the long-term survival under these adverse conditions. Thus, the crucial preadaptation for the successful colonisation of solid land was probably the ability to reorganise cellular structure in response to abiotic stress.

The contribution by Gorelova et al. (2015) leads to even more exotic habitats: The work reports on novel microalgae that have been isolated from the White Sea in association with different invertebrate hosts including sponges, polychaete worms, and hydrozoa. The cellular details of these microalgae match those described for the genus Desmodesmus (Nilshammar and Walles 1974). These coccal green algae belong to the most common and widespread inhabitants of fresh water ecosystems but also can settle in brackish water, such as the Baltic Sea. By molecular phylogeny and analysis of their surface ultrastructure, the authors can show that these newly discovered organisms really fall into this taxon, and they can group these endosymbionts into a monophyletic group, although they inhabit non-related hosts. The advantage of this (still facultative) symbiosis with an animal host seems to be in 
the ability to settle in otherwise non-accessible, saline, marine habitats and represents a further example for the adaptive versatility of the primitive unicellular algae.

\section{References}

Bakker ME, Lokhorst GM (1987) Ultrastructure of mitosis and cytokinesis in Zygnema sp. (Zygnematales, Chlorophyta). Protoplasma 138: $105-118$
Gorelova O, Baulina O, Solovchenko A, Chekanov K, Chivkunova O, Fedorenko T, Lobakova E (2015) Similarity and diversity of the Desmodesmus spp. microalgae isolated from associations with White Sea invertebrates. Protoplasma, current issue

Herburger K, Lewis LA, Holzinger A (2015) Photosynthetic efficiency, desiccation tolerance and ultrastructure in two phylogenetically distinct strains of alpine Zygnema sp. (Zygnematophyceae, Streptophyta): role of pre-akinete formation. Protoplasma, current issue

Nilshammar M, Walles B (1974) Electron microscope studies on cell differentiation in synchronized cultures of the green alga Desmodesmus. Protoplasma 79:317-332 